ON THE MAGELlANIC STREAM, THE MASS OF THE GALAXY AND THE AGE OF THE UNIVERSE

\author{
D. Lynden-Be11 \\ Institute of Astronomy, \\ The Observatories, \\ Cambridge CB3 OHA, England.
}

The Magellanic stream has been fitted with high accuracy in both position and velocity by the tidal tearing of a Magellanic Cloud. To get the good fit to the high velocity at the stream's tip at a suitable distance from the Galaxy we need either a large mass for the Galaxy, or a large circular velocity for the Sun, or both. An extragalactic method of determining the circular velocity yields the high value of $\mathrm{V}_{\mathrm{c}}=294 \pm 42 \mathrm{~km} / \mathrm{sec}$ and an orbit of poor accuracy for the relative motion of the Galaxy and the Andromeda nebula. Very large masses are needed if Andromeda and the Galaxy were formed together. A new direct determination of Hubble's constant from the "superluminal" expansion observed in VLB radio sources gives an age of the Universe of 9 billion years. Either larger masses still or smaller distances within the local group are necessary to bring Andromeda back towards us in so short a time.

Discovery of optical objects fying in the direction of the Magellanic Stream and other hydrogen clouds stimulated my interest in explaining the stream. Work by Hartwick and Sargent ${ }^{(2)}$ has since shown that the apparent coincidences are caused by projection since the velocities of the optical objects differ from those of the hydrogen. Nevertheless over the intervening years D.N.C. Lin and $I$ have run some forty thousand test particle orbits comprising 200 passages of a Magellanic Cloud clothed with 200 test particles 3 . Typically we start our Magellanic Cloud at apo-galacticon with its rotation inclined at some specified angle to the plane of the Magellanic Cloud's orbit. As our Magellanic Cloud sweeps past perigalacticon and on, it is tidally torn making a bridge towards the Galaxy and a tail away from it. We have discovered orbits which give a tail and no bridge and others that give bridges without tails. We have been at pains to fit the rather straight run of velocity with angle around the sky which is observed in the Magellanic Stream. We find this straightness to be a characteristic of orbits that are somewhat retrograde in the sense that the spin of the Magellanic Cloud has an inclination of $110^{\circ}$ to $130^{\circ}$ to its orbital angular momentum about the Galaxy. A description of the orbits searched over can be found in our paper. We search in dimensionless units and when we 


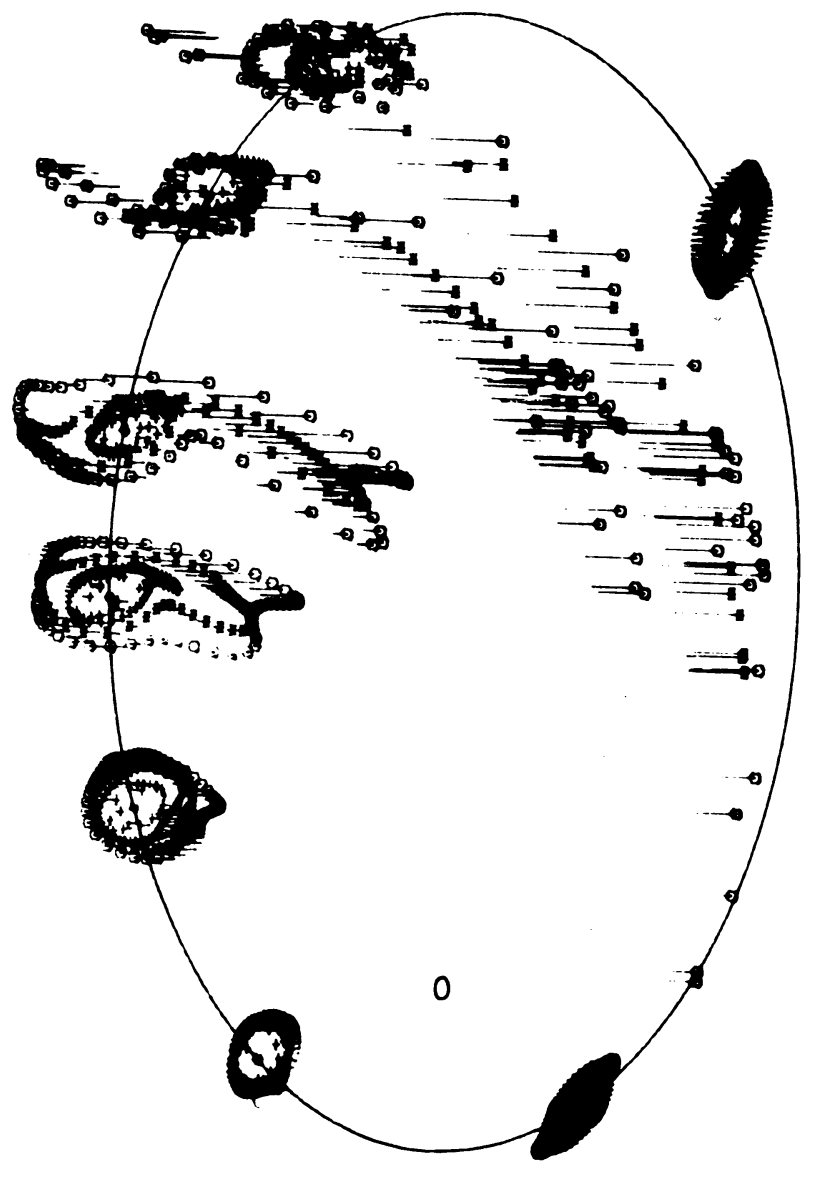

4

$\circ \propto$

.

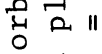

$+2$

두 क

荦望

ه

स्

30

रे है

ชెం

范 $\mathrm{m}$

(1) $\frac{1}{\infty} \underset{\infty}{\infty}$

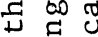

๘

כ)

तै हू

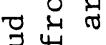

号 咅

บ 䒕 مै

- $\rightarrow$

先

가마

की

$\sum^{\infty}$

(1). U ل

बै

मू.

4 is

\已 ह

त्र

0 \& 1

$\infty$ 兵 20

.

노

Ш 4 II

计 है

ज

๑) ल

岁.

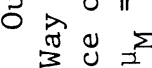

- 入

-1전

ब. न्न थ ल

败啠

- o \& \& II

的年. 
finally find an eccentricity e, a mass ratio $\mu_{M}$, a Magellanic Cloud size $r$, an inclination $i$ and a direction of spin at apogalacticon $P$ that can scale exactly onto the observations, we determine the length and velocity scales. Using Newton's gravitational constant G, we can then determine the mass of the Galaxy. We found that such a fit was not unique unless a circular velocity for the Sun's motion around the Galaxy was first specified; however a single best fit configuration did well in all cases if the velocity scale (and therefore mass scale) was changed for each choice of circular velocity assumed. (See Figure 1.)

To explain the high velocity of approach towards the Sun of the tip of the Magellanic stream we need either a large velocity for the Sun around the Galaxy so that there is less to explain in terms of infall, or a large infall velocity. A large infall velocity can arise either because the tip of the stream has been pulled inwards by a very heavy mass, or because the tip has fallen very deep into the Galaxy's potential. We found that the almost great circle shape of the stream was spoiled by parallax if the tip of the stream was too close to the Galaxy. We could not fit our best model to the positions of the stream on the sky if the tip was closer than about $16 \mathrm{kpc}$ from the galactic centre.(Davies and Wright ${ }^{4}$ have assumed that the stream's tip is a factor of two closer.) For the following circular velocities we get the following masses for the Galaxy:

$$
\begin{array}{lccc}
\mathrm{V}_{\mathrm{c}} / \mathrm{km} / \mathrm{sec} & 300 & 290 & 250 \\
\mathrm{M}_{\mathrm{G}} / \mathrm{M}_{\mathrm{O}} & 3.8 \cdot 10^{11} & 4.3 .10^{11} & 7.6 .10^{11}
\end{array}
$$

It is evident that unless the circular velocity is very large, then the Galaxy must have a heavy halo. This result turned our interest towards the value of the circular velocity.

The radial velocities of the galaxies in the local group reflect the motion of the observer which is the compound of the motion of our galaxy with the Sun's motion within it. It has always been assumed that these two velocities cannot be separated by extra-galactic measurements so that the speed of the Sun's circular motion about the galaxy $\mathrm{V}$ cannot be determined this way. However this is false. First dêtermine the Sun's motion, U, with respect to the galaxies of the local group excluding the Andromedá subgroup. Assuming these minor galaxies define the same zero of velocity as the centre of mass of the Galaxy and Andromeda, the momenta of the Galaxy and Andromeda must be equal and opposite. Thus if $\mathrm{G}$ is the galaxy's velocity, Andromeda's will be $-\mu G$, where $\mu$ is the mass ratio $M_{G} / M_{A}$. The observed radial velocity of Andromeda will therefore be

$$
\mathrm{v}_{\mathrm{Ar}}=(-\mu \underset{\sim}{\mathrm{G}}-\underset{\sim}{\mathrm{U}}){\underset{\sim}{\hat{\mathrm{A}}}}_{\mathrm{A}}=\left(-\mu\left(\underset{\sim}{\mathrm{U}}-\mathrm{V}_{\mathrm{C}} \underset{\sim}{\hat{\mathrm{C}}}\right)-\underset{\sim}{\mathrm{U}}\right) \cdot \stackrel{\hat{r}}{\mathrm{~A}}_{\mathrm{A}}
$$

where $\hat{\subseteq}$ is the direction of the circular motion and ${\underset{\sim A}{\hat{A}}}_{\text {is }}$ unit vector towards Andromeda, from which we may deduce 


$$
\mathrm{V}_{\mathrm{c}}=\left(\mathrm{v}_{\mathrm{Ar}}+(1+\mu) \underset{\sim}{\mathrm{U}} \cdot \underset{\sim}{\hat{c}}\right) /\left(\mu \stackrel{\hat{c}}{\mathrm{c}} \cdot \stackrel{\sim}{r} \mathrm{~A}_{\mathrm{A}}\right)
$$

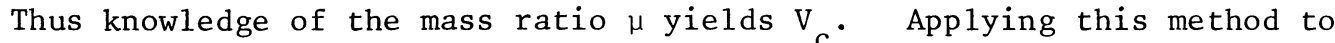
10 independent members of the local group yields $\mathrm{V}=294 \pm 42 \mathrm{~km} / \mathrm{sec}$ for the yelocity of free circular motion at the Sun's distance from the centre ${ }^{5}$. The dependence on $\mu$ has been removed by using the relationship of Tully \& Fisher which gives the luminosity and thus the mass of a spiral galaxy proportional to the $5 / 2$ power of its maximum circle velocity. With Andromeda's circular velocity at $265 \mathrm{~km} / \mathrm{sec}$ the least squares result is insensitive to $\mu$ in the range $\frac{1}{2}$ to 1.25 and the most probable result is always close to $290 \mathrm{~km} / \mathrm{sec}$.

This method yields not only $V$ but also $U$ and hence the Galaxy's velocity $G=\underline{U}-V_{c} \underset{\sim}{\hat{c}}$ and Andromedă's velocity $-\mu G$. With the aid of these we can calculate orbits for the galaxies about one another. The total mass $M_{A}+M_{G}$ required $(5)$ to bring them close to one another $2 \times 10^{10}$ years ago is $>1.3 \times 10^{12} \mathrm{M}$. A smaller circular velocity will yield a yet larger mass. My recent interpretation of "superluminal" expansion in VLB gives directly a Hubble constant of $110 \pm 10 \mathrm{~km} / \mathrm{sec} / \mathrm{Mpc}$, and a maximym timescale of only $9 \times 10^{9}$ years for the age of the Universe $(7,8)$. Unless the distance to the Andromeda nebula has been seriously over-estimated (by a factor of 1.5 or more) then the mass needed to bring the two galaxies together in so short a time as $9 \times 10^{9}$ years will be of the order of $4 \times 10^{12} \mathrm{M}$. However there is weak evidence that other galaxies, Maffei I in particular, intervened to complicate our naive picture of binary dynamics.

The Age of the Universe

Figure 2 shows VLB observations ${ }^{(9)}$ of the separation of components in the radio galaxy 3C120. They were originally interpreted (using a Hubble constant $\mathrm{H}_{0}=55 \mathrm{~km} / \mathrm{sec} / \mathrm{Mpc}$ ) as a burst expanding at $5 \mathrm{c}$ followed by another expanding at $8 \mathrm{c}$. However, the rediscussion that follows shows that a single expansion along the dotted line at $4.6 \mathrm{c}$ on that scale, accompanied by a rebrightening of a "central" object is a better interpretation. We picture the geometry involved as a flash of very strong waves in a tube formed by an accretion vortex about a black hole. This flash propagates both ways along the vortex tube and escapes as two equal pulses of strong waves, each travelling outwards at the velocity of light. The electric fields of each strong wave pulse accelerates the electrons it encounters and causes them to radiate synchro-compton radiation in the radio region. It is the regions currently exciting electrons that are seen as radio bright. Although the material of the sources is not moving rapidly outwards the excited patches move out with the velocity of light. If the two pulses set out an an angle $\theta$ to the line of sight, then the one moving towards the observer $\mathrm{S}_{\mathbf{l}}$ will move with apparent lateral speed $\underline{c} \sin \theta$ (1)with respect to central black hole 0 , while the other source $S_{2}^{-}$wili $\stackrel{\theta}{\text { move with apparent lateral speed } \frac{c \sin \theta}{1+\cos } \theta}$ (2) The apparent lateral separation velocity of $\mathrm{S}_{1}$ and $\mathrm{S}_{2}$ will thus be $v_{s}=\frac{2 c}{\sin \theta}$ (3)while the ratio of the separations will be $\frac{1-\cos \theta}{1+\cos \theta}$ (4) 


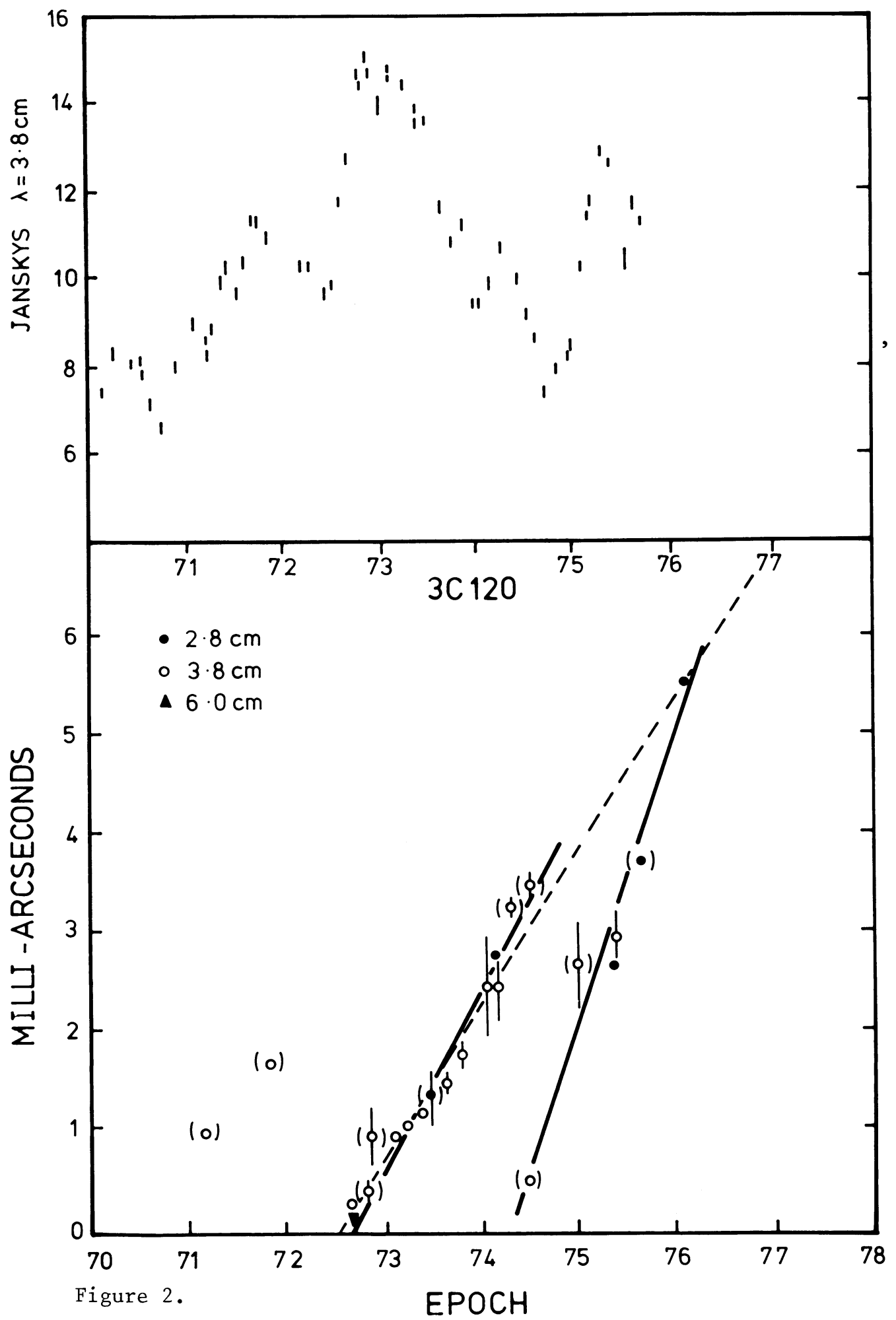


Application to $3 \mathrm{C} 120, \mathrm{z}=.033$

Figure 2 shows the observations given in reference 10 . The $3.8 \mathrm{~cm}$ flux shows an outburst in 1973 which caused a double source. This was seen as a simple double expanding at about $\mathrm{v}=5 \mathrm{c}$ (solid line) until 1974.2 when the double model failed to fit well, consequent upon a small increase of flux. In 1975 there followed a violent increase of flux and immediately a two source model fitted well again, but at about the same separation as it was at 1974.0 . The source began to dim in late 1975 and in 1976.1 it was again a we11-determined double although the flux at this time is not given. The authors of paper 10 have interpreted these events as the two superluminal expansions of $5 \mathrm{c}$ and $8 \mathrm{c}$ given by the heavy straight lines. However on my picture this would involve the vortex tube up the rotation axis of the radio galaxy to turn between the two events and I consider this most unlikely, especially as the observed position angle of separation on the sky are the same for both doubles. The following interpretation of events is more natural to me. $\quad 1972.5$ - 1973 a double expanding source $\mathrm{S}_{1}, \mathrm{~S}_{2}$ was born at 0 and expanded throughout 1973 as its flux diminished. '2 In early 1974 the object at 0 brightens, confusing the neat double interpretation of the data. In 1975.2 0 has increased in brightness so much that it forms a double with $\mathrm{S}_{1}$, the source moving towards us and $\mathrm{S}_{2}$ is outshone and ignored. 0 dims in late 1975 leading to a confused ${ }^{2}$ picture with $\mathrm{S}_{1}$, 0 and $S_{2}$ all seen. Finally in 19760 dies away leaving the old expanding source $\mathrm{S}_{1} \mathrm{~S}_{2}$. It is noted that this latest point fits on the dotted expansion line $(4.6 \mathrm{c})$ of the old source, which is a perfectly good alternative to the full line $(5 c)$ if one ignores confused data. The peculiar interest of this new interpretation arises because it not only removes the discrepancy of two different superluminal velocities observed from the same object, and therefore probably at the same $\theta$ but, because we see 0 at times, we can measure the ratio of the separations $\mathrm{OS}_{1}$ to $\mathrm{OS}_{2}$ and hence determine $\theta$. With $\theta$ determined, the apparent transverse separation velocity is known from (3) so the observed angular velocity gives the distance and hence Hubble's constant. The small z=0.033 of $3 \mathrm{C} 120$ makes the cosmological corrections unimportant for this source, so the Hubble constant derived in this case is independent of $q_{0}$. However the same method applied to 3C 345 should yield a reasonably good value of $\mathrm{q}_{\mathrm{o}}$ too.

If $\theta$ were $90^{\circ}$ the clear double seen in 1975.4 would have been half way up to the dotted line. Its fractional offset $\left(0 \mathrm{~S}_{1}-\frac{1}{2} \mathrm{~S}_{1} \mathrm{~S}_{2}\right) / \mathrm{S}_{1} \mathrm{~S}_{2}$ corresponds to the offset of 0 from the centre of $S_{1}$ and $\mathrm{S}_{2}$ and $1 \mathrm{~s}^{2}$ therefore just $\frac{1}{2} \cos \theta$. From this we find $\theta=68^{\circ l_{ \pm}} 10^{\circ}$. It is $^{2}$ is particularly interesting that this angle is close to the inclination of the apparent disk of 3C120 measured from Arp's picture on which it appears to be a disturbed spiral. Measuring his picture we get $i=55^{\circ}-60^{\circ}$ but this may well be in error by $10^{\circ}$ due to the fact that the disturbed disk does not have a circular outline. Furthermore the observed separation of the radio double, although fairly close to the apparent mincor axis of the optical image is not along it. It seems reasonable to assume that ejection is taking place along the rotation axis, but the 
galaxy has imperfect circular symmetry. As in such a case $i$ should equal $\theta$ and both determinations have similar errors we shall adopt $\theta=64 \pm 8^{\circ}$. The light echo theory then gives us

$$
\mathrm{v}_{\mathrm{s}}=2 \mathrm{c} / \sin 64^{\circ}=2.22 \mathrm{c} \pm 0.19 \mathrm{c}
$$

The authors of reference 5 adopt $\mathrm{H}=55 \mathrm{~km} / \mathrm{sec} / \mathrm{Mpc}$, and obtained $5 \mathrm{c}$ for this velocity, but our correction to the dotted line interpretation of Figure 2 yields $4.6 \mathrm{c}$ on that scale. To get the answer deduced from the light echo theory we must change the Hubble constant by a factor $\underline{4.6 \pm .2}=2.1 \pm 0.2$. We deduce a Hubble constant of $115 \pm 12 \mathrm{~km} / \mathrm{sec} /$ $2.22 \pm .19 \quad$ Mpc. Similar work on the $3 \mathrm{C} 273$ observations yields $\theta=79^{\circ}$ and a Hubble constant of $113 \pm 10 \mathrm{~km} / \mathrm{sec} / \mathrm{Mpc}$. The statistics of the superluminal expansions of $\mathrm{S}_{1} \mathrm{~S}_{2}$ for $3 \mathrm{C} 120,3 \mathrm{C} 273$ and $3 \mathrm{C} 345$ yields $\mathrm{Ho}=110 \pm 20 \mathrm{~km} / \mathrm{sec} / \mathrm{Mpc}$. In summary this physical method of measuring $\mathrm{H}$ yielgs $\mathrm{Ho}=110 \pm 10 \mathrm{~km} / \mathrm{sec} / \mathrm{Mpc}$ and a maximum age of the Universe $\mathrm{H}_{\mathrm{O}}^{-1}=(9 \pm 1) \times 10^{9}$ years provided the interpretation is correct.

References

1. Lynden-Be11, D.: 1976, Month1y Notices Roy.Astron.Soc. 174, p.695.

2. Hartwick, D., and Sargent, W.L.W.: 1977, preprint Astrophys.J.

3. Lind, D.N.C., and Lynden-Be11, D.: 1977, Month1y Notices Roy. Astron.Soc.

4. Davies, R.D., and Wright, A.E.: 1977, Monthly Notices Roy. Astron.Soc. 180, p.71.

5. Lynden-Be11, D., and Lin, D.N.C.: 1977, Monthly Notices Roy. Astron. Soc. 181, p.

6. Tully, R.B., and Fisher, J.R.: 1977, Astron.Astrophys.54, p.661.

7. Lynden-Be11, D.: 1977, Nature in press.

8. Lynden-Be11, D.: 1977, Proc. of the Cambridge NATO Summer School (editors S. Mitton and C. Hazard), on Quasars.

9. Cohen, M.H.: 1977, Nature, 268, p.405.

10. Cohen, M.H., Linfield, R.P., Moffet, A.T., Romney, J.D, Seielstad, G.A., Kellermann, K.I., Shaffer, D.B., Pauliny-Toth, I.I.K., Preuss, E., Witze1, A., Schilizzi, R.T., Geldzahler, B.J. 1977, Nature, 268, p.405.

\section{DISCUSSION}

de Vaucouleurs: Do you believe that the spin axis of the accretion disk in the black hole model coincides with the spin axis of the galactic disk?

Lynden-Belz: If the system has been left alone without interaction for a long time, then yes.

de Vaucouleurs: If so, does $\theta=68^{\circ}$ agree with the inclination derived from the shape of $3 \mathrm{C} 120$ ? 
Lynden-Bezl: $\quad i=55^{\circ}-60^{\circ} \pm 10^{\circ}$

$\theta=68^{\circ} \pm 10^{\circ}$

I think this is good agreement. I took $\theta=64^{\circ}$.

\title{
THE STELLAR COMPONENT OF THE MAGELLANIC STREAM
}

\author{
Wil1iam E. Kunke1
}

The problem of the Magellanic Stream (MS) is re-examined considering also stellar systems (globular clusters and dwarf spheroidal galaxies) lying between 25 and $250 \mathrm{kpc}$ from the Galactic center (Kunke1 and Demers 1976). The distribution of these systems is shown to be nonrandom, with a planar component whose inclination to the Local Group is $43^{\circ}$, and to the local supergalaxy exceeds $60^{\circ}$. The velocity distribution of members of the group shows a symmetry typical of debris associated with a disruptive event at perigalacticon of a victim moving in an elliptical orbit.

These data are examined in terms of tidal disruption affecting the Magellanic Cloud system, with a view to constraining the multidimensional character of admissible solutions from modelling efforts using computer simulation. The observed angle between the plane of the MS and that of the stellar or Magellanic Plane Group (MPG) is explained in terms of one being residue from a bridge and the other from a tail. The angle depends to a first approximation on only the mass ratio of the victim to the perturber, which is found to be 0.028 , consistent with classical estimates for the masses of the LMC and the Galaxy.

Color-magnitude studies of the stellar systems, as well as the marked composition difference between the MS and the Magellanic Clouds point to a considerable age for the epoch of the most disruptive event, possibly just less than the age of the Galactic globular cluster system.

The sense of orbital motion (which also determines the status of bridge or tail) is determined independently from the age of the oldest (and most numerous) stellar component in the Wing of the Smal1 Cloud, from the dominance of HI in the MS and of stars in the MPG, and from the uniformity (or its lack) of debris material in position along the debris orbits.

The model most favored by these arguments moves in a sense opposite to that of Davies and Wright (1977), and of Lin and Lynden-Be11 (1977). The first encounter producing the most pronounced disruption (and much of the MPG and MS) occurred some time ago; the current position of the Magellanic Clouds near perigalacticon correspond to a second or possibly subsequent encounter with relatively mild interaction; the orbital period is of the order of $5 \times 10^{9}$ years. The total Galactic mass lying inside perigalacticon consistent with this model is a factor of 3 or 4 greater than the classical values in current use. 